Egyptian Journal of Aquatic Biology \& Fisheries

Zoology Department, Faculty of Science,

Ain Shams University, Cairo, Egypt.

ISSN $1110-6131$

Vol. 25(3): 1027 - 1043 (2021)

www.ejabf.journals.ekb.eg

\title{
Abundance and distribution of zooplankton communities inhabiting the intertidal zone of the Suez Gulf, Red Sea, Egypt
}

\author{
Aldoushy Mahdy ${ }^{1}$, Hesham El-Abd ${ }^{2}$ * and Mohamed Hamza Hasan ${ }^{2}$ \\ 1. Department of Zoology, Faculty of Science, Al-Azhar University (Assiut Branch), Egypt. \\ 2. Department of Aquatic Ecology, Faculty of Fish Resources, Suez University, Suez, Egypt. \\ "Corresponding Author: Heshamelabd508@yahoo.com
}

\begin{abstract}
ARTICLE INFO
Article History:

Received: July 5, 2021

Accepted: July 17, 2021

Online: Aug. 11, 2021

Keywords:

Abundance,

Distribution,

Zooplankton,

Copepoda,

Gulf of Suez,

Red Sea,

Egypt

ABSTRACT

Zooplankton communities at the Gulf of Suez were investigated during the current study and their abundance was correlated with the physico-chemical parameters of the area. The goal of the study was to estimate the distribution and abundance of zooplankton taxa under various habitat stressors, and update the ecological data base of the study area. The study recorded 39 species of zooplankton in 16 taxa with total abundance of 66300 organisms across all investigated sites. The organisms were distributed between holoplankton that recorded approximately 55560 organisms $(84.2 \%)$ and the meroplankton which recorded approximately 10740 organisms $(15.8 \%)$. The most abundant groups were Copepoda 52340 organisms (78.9\%), larval stages (11\%) and Maxillopoda (3\%). While, Anthomedusea, Leptomeduea, and Pteropoda were rarely recorded. Among the surveyed sites, Zafarana recorded the highest abundance (23537 Organisms) with a percentage of 63 of the total zooplankton populations, whereas the lowest site in abundance was Port-Tewfik which recorded (6656 organisms), represented a percentage of $10 \%$ of the total populations. The current work showed that copepods recorded a significant difference in spatial distribution, while copepods, dissolved oxygen, water temperature and total dissolved solids differed significantly at temporal distribution.
\end{abstract}

\section{INTRODUCTION}

The geological structure and geographical position of the Red Sea have a remarkable influence on zooplankton communities. The Red Sea is a flooded valley that can be described as a young ocean, created by pulling a part of Africa and Arabia. It is a North-South elongation extending from $12.5^{\circ} \mathrm{N}$ in the south to $30^{\circ} \mathrm{N}$ at Suez in the north (Sofianos \& Johns, 2002). It includes the Gulf of Aqaba, the Gulf of Suez and the Red Sea proper (Thurman, 1997). Red Sea is characterized by high temperature and salinity as well as oligotrophic conditions (Raitsos et al., 2013).

Few studies were conducted on the diversity of zooplankton in the Red Sea. These studies are rare limited to specific locations in the northern Red Sea and Gulf of Aqaba (Echelman \& Fishelson, 1990; Khalil \& Abdel-Rahman, 1997; Cornils et al., 2007; El-Sherbiny et al., 2007). Eminently, the Red Sea is ideal for studying the distribution and diversity of zooplankton communities. Zooplankton serve as a vital link between phytoplankton and higher trophic levels in the marine food chain such as fishes and 
whales (Wyatt et al., 2012). Zooplankton are abundant in all of the world's oceans, seas, and lakes, and their abundance varies horizontally, vertically and seasonally. The primary source of this variability is the phytoplankton availability and the secondary one is the availability of light (Thurman, 1997). Zooplankton play crucial roles in marine biogeochemical cycles and food webs, acting as a link for energy transfer from lower to higher trophic levels. Moreover, they have a fundamental role in the recycling and mediating of macronutrients (Mitra et al., 2014) Zooplankton communities usually vary in composition as certain species are highly sensitive to changes in temperature, nutrient cycling, and environmental fluctuations (Primo et al., 2015).

Changes in zooplankton community composition can affect the degree of up and down regulations of phytoplankton communities, influence the amount of nutrient availability and processing, and can determine the capacity of aquatic ecosystems as well (Brucet et al., 2010). Some biotic and abiotic parameters, such as temperature, habitat differences, the presence or absence of fish and macrophytes may influence the richness and composition of zooplankton species (Kaya et al., 2010). Moreover, the distribution of zooplankton is useful for assessing environmental issues, such as eutrophication, warming trends, pollution and hydrographic events (Michael, 1984; ObuidAllah et al., 2005), which are very relevant for other biological and ecological researches that require investigation at the Gulf of Suez.

Diversity of zooplankton in the Gulf of Suez seems to be very high where most of the major zooplankton taxa are represented. Tintinnids, foraminifera, copepods, hydromedusa as well as chaetognatha are found in high abundance while other meroplankton groups, such as shrimp larvae, crab larvae, crustacean larvae, mollusks larvae, and chordates larvae were also recorded in large numbers (Abu El-Regal $\boldsymbol{e t}$ al., 2018). The most abundant taxa in the Gulf region was Copepoda which formed 78-92\% of all zooplankton groups (Abdel-Rahman, 1993; Dorgham et al., 2012). Additionally, Aboul Ezz et al. (2014) found that copepods constituted about $72 \%$ of the total zooplankton collected from Matrouh beaches of the Mediterranean Sea.

The present study seeked to estimate the distribution and abundance of zooplankton qualitatively and quantitatively with a list of recorded species in different sites of the Gulf of Suez and determine the role of physicochemical parameters in zooplankton abundance in the Gulf.

\section{MATERIALS AND METHODS}

\subsection{Study Sites}

Five sites were investigated along the Gulf of Suez, these were Port-Tewfik (site 1), Cabanon (site 2), National Institute of Oceanography and Fisheries, Suez Branch (NIOF) (site 3), Ain-Sokhna (site 4), and Zafarana (site 5) (Fig.1). Port-Tewfik: affected mainly by oil pollution and wastes from shipping operations at Port-Tewfik port. Cabanon: affected mainly by sewage pollution. NIOF: affected mainly by the higher temperature from the cooling water of Ataqa power plant and also by sewage pollution. Ain-Sokhna: affected mainly by tourism and human recreational activities. Zafarana: is a calm area that has the lowest influence by human activities (Fig.1). 


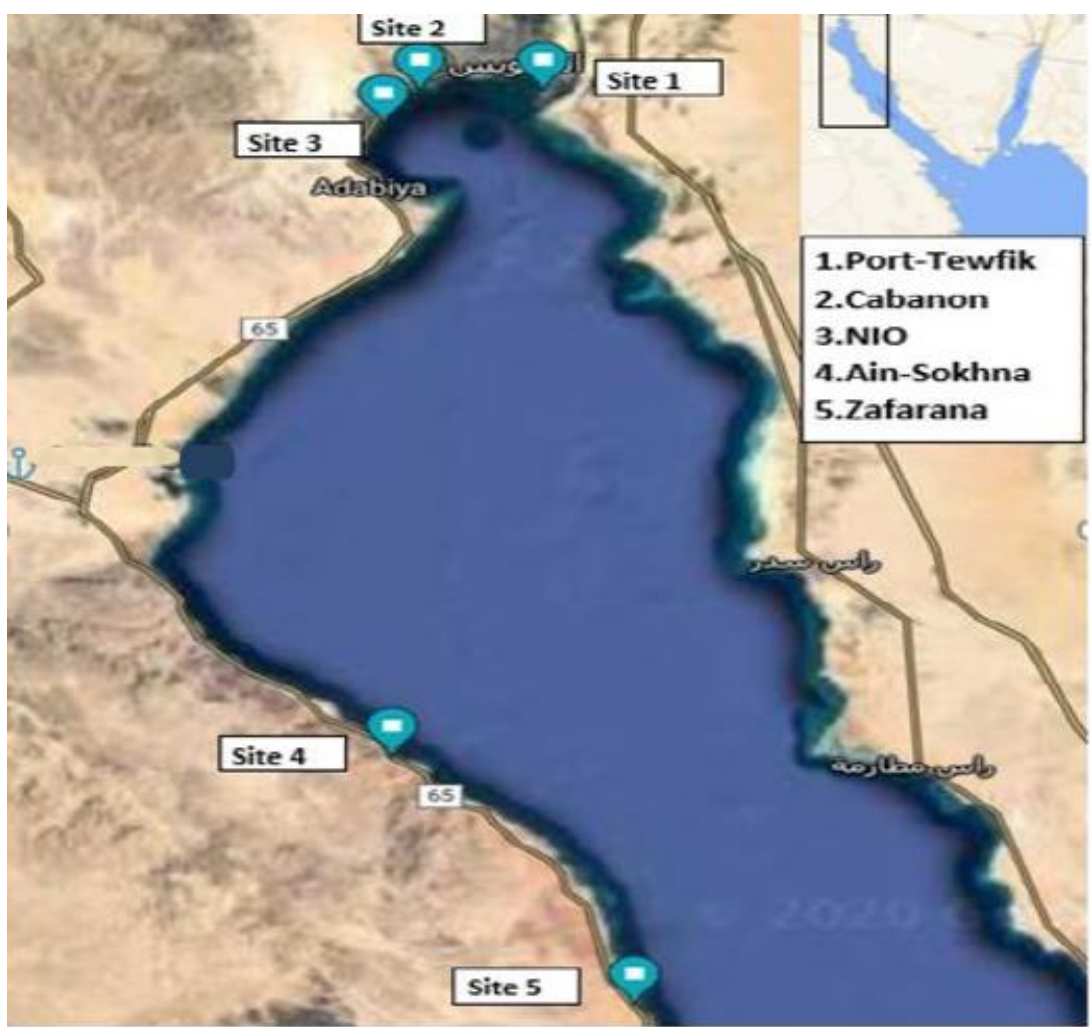

Fig. 1. A map showing study sites in the Gulf of Suez, Red Sea, Egypt.

\subsection{Sample collection}

Zooplankton samples were collected seasonally from January to November 2019 from the intertidal zone of the Gulf of Suez. Data of date, time, human activities and physico-chemical parameters were collected. For quantitative study; the zooplankton samples were collected by filtering 300 litres of the seawater (collected by plastic bucket) through a fine zooplankton net at each site. The plankton net has $100 \mu \mathrm{m}$ mesh size with a mouth diameter of $44 \mathrm{~cm}$ and a total length of $100 \mathrm{~cm}$. For qualitative samples; plankton net was towed randomly at each site. The zooplankton organisms retained in the net were transferred carefully into suitable plastic bottles, labeled and immediately fixed with 5\% neutral formalin solution after measuring the displacement volume. After each haul, the net was washed thoroughly with seawater and the organisms retained were added to the collected sample to prevent any loss of the hauled organisms (Al-Yamani \& Skryabin, 2006).

In the laboratory, the collected samples were allowed to stand for sufficient time in the graduated cylinders to ensure complete settlement of the zooplankton organisms and the surplus water of each sample was siphoned off until its volume equaled $100 \mathrm{ml}$. The whole sample was examined by placing in a large Petri dish, and the large organisms such as fish larvae were picked and counted. Sub-sample of $5 \mathrm{ml}$ was then transferred into a counting chamber and each plankter was counted separately using an inverted microscope Leitz, Wetzlar, Germany, Type 090-123, 012. For each sample, 3 sub- 
samples were estimated. The samples were preserved in $70 \%$ ethanol for long time fixation.

\subsection{Identification of zooplankton}

The separated zooplankton species were identified following the descriptive keys of Bradford-Grieve and Jillett (1980), Bradford-Grieve (1994), Heron and Bradford-Grieve (1995) and Al-Yamani et al. (2010). For accurate identification of copepods, each copepod was dissected with fine needles and mounted semi-permanently in ksiser's Glycerin jelly (Gatenby \& Painter, 1937). This mixture was set when cooled but may be melted by gentle heat. Thus, a limb or a whole animal may be observed from different angles. All small or delicate copepods were first left in a mixture of glycerin, alcohol, and water in the proportions 1:1:2, respectively, until the mixture was concentrated, otherwise the integument would collapse and distort the copepod form. The magnitude of the standing crop of zooplankton was calculated as the numbers of the different species per cubic meter in all samples (Individual $/ \mathrm{m}^{3}$ ).

The recorded zooplankton taxa were divided into constancy classes according to the system adopted by Abd El-Wakeil (2005) as follows: Constant taxa: present in more than $50 \%$ of the samples; accessory taxa: present in 50-25\% of the samples and accidental taxa: present in less than $25 \%$ of the samples.

\subsection{Measurement of physico-chemical parameters}

Seasonally measurements of water temperature $\left({ }^{\circ} \mathrm{C}\right)$, hydrogen ion concentration $(\mathrm{pH})$, dissolved oxygen (DO) (mg/l), and total dissolved solids (T.D.S) (\%o) were carried out in the field by using Water Quality Checker.

\subsection{Statistical analysis}

Analysis of variance on Statistical Package for Social Science (SPSS) version 22 (2014). (SYSTAT statistical program) was used to test the present data. In the case of significant difference, the Multiple Range Comparison (Least significant difference; LSD) was selected from the PostHoc window on the same statistical package to detect the distinct variance among means. In addition, Pearson correlation coefficient and multiple regressions were applied in the present data.

Probability value $\leq 0.05$ was defined as significant throughout the present study, and the value $>0.05$ was defined as non-significant and that less than 0.01 was defined as highly significant.

\section{RESULTS}

\subsection{Species diversity}

The current study recorded a total number of 39 species; larvae of 9 groups and Nauplius of two groups. The larvae and Nauplii were distributed at 16 taxa. These taxa were 7 holoplankton groups (Tintinnidea, Foraminifera, Anthomedusea, Leptomeduea, Pteropoda, Copepoda, Chaetognatha) that include the highest abundant groups which represented $84.2 \%$ of the total populations with 55846 individuals, followed by 9 
meroplankton groups (Polychaeta, Maxillopoda, Decapoda, Bivalvia, Gastropoda Urochordata, Echinodermata Cephalochordata and Chordata) that represented $15.8 \%$ of the zooplankton populations at the investigated sites with abundance of 10454 individuals (Table 1).

Table 1. Total number and percentage (\%) of zooplankton taxa at different sites during investigated period.

\begin{tabular}{|c|c|c|c|c|}
\hline NO. & Zooplankton type & Zooplankton Taxa & Abundance & $\mathrm{P}(\%)$ \\
\hline 1 & \multirow{7}{*}{ Holoplankton } & Tintinnidea & 1016 & 1.5 \\
\hline 2 & & Foraminiferida & 1413.0 & 2.1 \\
\hline 3 & & Anthomedusea & 140.0 & 0.2 \\
\hline 4 & & Leptomeduea & 146.7 & 0.2 \\
\hline 5 & & Pteropoda & 407 & 0.6 \\
\hline 6 & & Copepoda & 52340.0 & 78.9 \\
\hline 7 & & Chaetognatha & 383.3 & 0.6 \\
\hline \multicolumn{3}{|c|}{ Total Holoplankton } & 55846 & 84.2 \\
\hline 8 & \multirow{9}{*}{ Meroplankton } & Urochordata & 1576 & 2.4 \\
\hline 9 & & Polychaeta & 1524 & 2.3 \\
\hline 10 & & Bivalvia & 593 & 0.9 \\
\hline 11 & & Gastropoda & 3907 & 5.9 \\
\hline 12 & & Maxillopoda & 2007 & 3.0 \\
\hline 13 & & Decapoda & 279 & 0.4 \\
\hline 14 & & Echinodermata & 110 & 0.2 \\
\hline 15 & & Cephalochordata & 34 & 0.05 \\
\hline 16 & & Chordate & 424 & 0.6 \\
\hline \multicolumn{3}{|c|}{ Total Meroplankton } & 10454 & 15.8 \\
\hline \multicolumn{3}{|c|}{ Total zooplankton taxa } & 66300 & 100 \\
\hline
\end{tabular}

Copepoda was represented by 26 species plus two larval stages. Tintinnidea and Urochordata were represented by 3 species. Chaetognatha and Maxillopoda were represented by 2 species. Foraminiferida, Anthomedusea, Leptomeduea and Pteropoda were represented by one species for each group. Decapoda was represented by 2 larvae. Polychaeta, Bivalvia, Gastropoda, Echinodermata, Cephalochordata and Chordate were represented by one species of larvae for each group (Table 3). Zafarana and Ain-Sokhna recorded the highest diversity among all sites surveyed recording 49 species/taxa and larvae. It was followed by NIOF that recorded 47 species/taxa and larvae, followed by Cabanon site that recorded 42 species/taxa and larvae while Port-Tewfik recorded 37 species/taxa and larvae that represented the lowest diversity (Table 2).

Species diversity analysis among different sites showed that site 1 differed significantly from all other sites $(\mathrm{P} \leq 0.01)$, and site 2 differed significantly from all other 
sites $(\mathrm{P} \leq 0.01)$, while there was no significant differences among (site 3,4 and 5) $(\mathrm{P}>$ $0.05)$ as presented in Table (2).

Table 2. Significant differences of total zooplankton diversity among different sites $(\mathrm{P} \leq 0.01)$. ( $a, b$ and $\mathrm{c}$ refer to the significant differences).

\begin{tabular}{|l|c|c|c|c|c|}
\hline Sites*diversity & Site 1 & Site 2 & Site 3 & Site 4 & Site 5 \\
\hline Site 1 & - & $* *$ & $* *$ & $* *$ & $* *$ \\
\hline Site 2 & $* *$ & - & $* *$ & $* *$ & $* *$ \\
\hline Site 3 & $* *$ & $* *$ & - & NS & NS \\
\hline Site 4 & $* *$ & $* *$ & NS & - & NS \\
\hline Site 5 & $* *$ & $* *$ & NS & NS & - \\
\hline Sites*diversity & $37 \mathrm{c}$ & $42 \mathrm{~b}$ & $47 \mathrm{a}$ & $49 \mathrm{a}$ & $49 \mathrm{a}$ \\
\hline
\end{tabular}

\subsection{Species density}

During the current study, zooplankton species showed both temporal and spatial variation among all species recorded. The most abundant species was Oithona nana that belongs to Copepoda. This species recorded the highest abundance during the study period at all sites (6471 individuals), the maximum density was recorded at Zafarana in summer $\left(1000 \mathrm{Ind} / \mathrm{m}^{3}\right)$ while it recorded the minimum density $\left(20 \mathrm{Ind} / \mathrm{m}^{3}\right)$ in winter at Ain-Sokhna. While, the lowest abundant species was Paracalanus indicus that belongs to Copepoda which recorded 19 individuals during the study period at all sites. The maximum density was recorded in summer at Zafarana $\left(7 \mathrm{Ind} / \mathrm{m}^{3}\right)$ while the lowest density was recorded in winter, autumn and spring at NIOF, Ain-Sokhna and Zafarana, respectively. Moreover, it was not recorded in other seasons at other different sites (Table 3).

The result showed that, the highest density was achieved at site 5 (23537 individuals), followed by site 4 (14803 organisms), then comes site 3 (12053 individuals), followed by site 2 (92500 individuals). While, the lowest density was recorded at site 1 with 6656 individuals (Table 4). The results showed that the total species density significantly differed among all sites of investigations $(\mathrm{P} \leq 0.01)$ (Table 4$)$. 
Table 3. Density of the recorded zooplankton taxa at different sites and seasons during the investigated period (Ind / $\mathrm{m}^{3}$ ). Wi: winter, Sp: spring,

Su: summer and Au: autumn.

\begin{tabular}{|c|c|c|c|c|c|c|c|c|c|c|c|c|c|c|c|c|c|c|c|c|c|}
\hline \multirow[b]{2}{*}{ Zooplankton taxa } & \multicolumn{4}{|c|}{ Site 1 Port-Tewfik } & \multicolumn{4}{|c|}{ Site 2 Cabanon } & \multicolumn{4}{|c|}{ Site 3 NIOF } & \multicolumn{4}{|c|}{ Site 4 Ain Sokhna } & \multicolumn{4}{|c|}{ Site 5 Zafarana } & \multirow{2}{*}{ Total } \\
\hline & $\mathrm{Wi}$ & $\mathrm{Sp}$ & $\mathrm{Su}$ & $\mathrm{Au}$ & $\mathrm{Wi}$ & $\mathrm{Sp}$ & $\mathrm{Su}$ & $\mathrm{Au}$ & $\mathrm{Wi}$ & $\mathrm{Sp}$ & $\mathrm{Su}$ & $\mathrm{Au}$ & $\mathrm{Wi}$ & $\mathrm{Sp}$ & $\mathrm{Su}$ & $\mathrm{Au}$ & $\mathrm{Wi}$ & $\mathrm{Sp}$ & $\mathrm{Su}$ & $\mathrm{Au}$ & \\
\hline \multicolumn{22}{|l|}{ COPEPODA } \\
\hline Nauplius larvae & 433 & 567 & 390 & 700 & 700 & 850 & 967 & 867 & 963 & 1060 & 1167 & 1000 & 1050 & 1150 & 1267 & 1533 & 1700 & 1833 & 1383 & 2290 & 21870 \\
\hline Copepodite stage & 233 & 323 & 400 & 317 & 317 & 493 & 667 & 383 & 333 & 450 & 700 & 317 & 333 & 350 & 667 & 577 & 1300 & 773 & 827 & 1400 & 11160 \\
\hline Paracalanus crassirostris & 3 & 67 & 73 & 7 & 17 & 43 & 67 & 20 & 33 & 70 & 87 & 7 & 0 & 40 & 117 & 217 & 153 & 100 & 107 & 143 & 1371 \\
\hline Paracalanus parvus & 0 & 0 & 0 & 0 & 0 & 0 & 0 & 0 & 3 & 0 & 0 & 0 & 0 & 0 & 0 & 50 & 0 & 67 & 10 & 150 & 280 \\
\hline Paracalanus indicus & 0 & 0 & 0 & 0 & 0 & 0 & 0 & 0 & 3 & 0 & 0 & 0 & 0 & 0 & 0 & 3 & 0 & 3 & 7 & 3 & 19 \\
\hline Acrocalkanus gibber & 0 & 37 & 23 & 40 & 0 & 23 & 17 & 30 & 0 & 23 & 0 & 30 & 0 & 17 & 33 & 33 & 50 & 100 & 0 & 100 & 556 \\
\hline Centropages furcatus & 3 & 7 & 0 & 10 & 3 & 3 & 0 & 7 & 7 & 3 & 7 & 10 & 3 & 10 & 3 & 0 & 10 & 0 & 0 & 0 & 86 \\
\hline Acartia negligens & 10 & 20 & 17 & 3 & 33 & 10 & 3 & 17 & 33 & 3 & 7 & 13 & 13 & 10 & 33 & 0 & 7 & 100 & 107 & 0 & 439 \\
\hline Acartia centrura & 0 & 10 & 0 & 3 & 0 & 7 & 7 & 3 & 0 & 3 & 17 & 3 & 0 & 10 & 17 & 0 & 43 & 7 & 0 & 17 & 147 \\
\hline Clausocalanus furcatus & 7 & 0 & 10 & 3 & 33 & 0 & 0 & 3 & 417 & 7 & 0 & 3 & 150 & 0 & 0 & 200 & 110 & 250 & 250 & 250 & 1693 \\
\hline Calanopia elliptica & 0 & 0 & 3 & 0 & 0 & 3 & 3 & 0 & 0 & 3 & 0 & 0 & 0 & 3 & 0 & 0 & 3 & 0 & 0 & 0 & 18 \\
\hline Calanus minor & 7 & 0 & 0 & 0 & 17 & 0 & 0 & 0 & 50 & 0 & 3 & 0 & 17 & 0 & 3 & 0 & 10 & 7 & 140 & 0 & 254 \\
\hline Mecynocera clause & 0 & 0 & 0 & 0 & 3 & 0 & 0 & 0 & 7 & 0 & 0 & 0 & 0 & 0 & 0 & 7 & 0 & 7 & 23 & 10 & 57 \\
\hline Oithona nana & 3 & 567 & 33 & 233 & 33 & 500 & 73 & 367 & 33 & 477 & 367 & 283 & 20 & 567 & 867 & 57 & 317 & 617 & 1000 & 57 & 6471 \\
\hline Oithona plumifera & 3 & 7 & 7 & 7 & 17 & 3 & 33 & 3 & 27 & 30 & 17 & 7 & 10 & 7 & 33 & 0 & 7 & 0 & 1003 & 0 & 1221 \\
\hline Oncaea media & 0 & 3 & 33 & 3 & 60 & 3 & 0 & 0 & 240 & 20 & 3 & 3 & 100 & 3 & 33 & 50 & 283 & 200 & 283 & 50 & 1370 \\
\hline Oncaea conifer & 0 & 0 & 0 & 0 & 0 & 0 & 0 & 0 & 0 & 0 & 0 & 0 & 0 & 0 & 0 & 0 & 0 & 27 & 0 & 0 & 27 \\
\hline Corycaeus erythraeus & 0 & 0 & 0 & 0 & 0 & 0 & 0 & 0 & 0 & 0 & 0 & 0 & 0 & 0 & 0 & 50 & 0 & 7 & 90 & 50 & 197 \\
\hline Corycaeus speciosus & 0 & 0 & 0 & 0 & 57 & 0 & 0 & 0 & 63 & 0 & 0 & 0 & 23 & 0 & 0 & 60 & 0 & 103 & 7 & 33 & 346 \\
\hline Euterpina acutifrons & 0 & 23 & 17 & 233 & 0 & 10 & 10 & 217 & 0 & 133 & 33 & 233 & 0 & 50 & 67 & 0 & 7 & 53 & 87 & 0 & 1173 \\
\hline Microsetella norvegica & 0 & 7 & 90 & 17 & 0 & 7 & 67 & 43 & 0 & 107 & 67 & 43 & 0 & 3 & 117 & 0 & 10 & 0 & 0 & 0 & 578 \\
\hline Clytemnestra scutellata & 0 & 0 & 17 & 27 & 0 & 0 & 83 & 17 & 0 & 0 & 83 & 10 & 0 & 0 & 133 & 0 & 0 & 0 & 0 & 0 & 370 \\
\hline Paracalanus aculatus & 0 & 0 & 40 & 3 & 0 & 0 & 83 & 7 & 0 & 0 & 67 & 3 & 0 & 0 & 200 & 0 & 37 & 0 & 0 & 0 & 440 \\
\hline Centropagus elongates & 0 & 0 & 0 & 0 & 3 & 0 & 17 & 0 & 17 & 0 & 10 & 0 & 0 & 0 & 17 & 17 & 0 & 3 & 3 & 83 & 170 \\
\hline Clausocalanus arcuicornisl & 10 & 0 & 0 & 0 & 20 & 0 & 0 & 0 & 100 & 0 & 0 & 0 & 23 & 0 & 0 & 33 & 0 & 7 & 3 & 50 & 246 \\
\hline Ctenocalanus vanus & 0 & 0 & 0 & 0 & 37 & 0 & 0 & 0 & 3 & 0 & 0 & 0 & 0 & 0 & 0 & 33 & 0 & 3 & 7 & 33 & 116 \\
\hline Farranula gibbula & 0 & 0 & 0 & 0 & 17 & 0 & 0 & 0 & 140 & 0 & 0 & 0 & 83 & 0 & 0 & 433 & 217 & 307 & 250 & 167 & 1614 \\
\hline Copilia mirabilis & 0 & 0 & 0 & 0 & 7 & 0 & 0 & 0 & 3 & 0 & 0 & 0 & 0 & 0 & 0 & 17 & 0 & 0 & 20 & 0 & 47 \\
\hline \multicolumn{22}{|l|}{ TINTINNIDEA } \\
\hline Tintinnopsis campanula & 27 & 30 & 43 & 7 & 23 & 93 & 43 & 20 & 20 & 63 & 40 & 7 & 37 & 70 & 57 & 23 & 50 & 53 & 57 & 23 & 786 \\
\hline
\end{tabular}




\begin{tabular}{|c|c|c|c|c|c|c|c|c|c|c|c|c|c|c|c|c|c|c|c|c|c|}
\hline Favella ehrenbergii & 7 & 0 & 7 & 0 & 10 & 7 & 3 & 0 & 0 & 23 & 7 & 0 & 3 & 3 & 10 & 10 & 23 & 7 & 10 & 13 & 143 \\
\hline Stenosemella ventricosa & 3 & 0 & 0 & 0 & 3 & 20 & 0 & 0 & 7 & 20 & 3 & 3 & 3 & 3 & 0 & 7 & 3 & 3 & 0 & 7 & 85 \\
\hline \multicolumn{22}{|l|}{ FORAMINIFERA } \\
\hline Globigerina inflate & 23 & 93 & 57 & 63 & 27 & 57 & 57 & 100 & 67 & 57 & 40 & 117 & 140 & 107 & 67 & 50 & 73 & 63 & 107 & 50 & 1415 \\
\hline \multicolumn{22}{|l|}{ Anthomedusea, } \\
\hline Podocoryne areolate & 3 & 0 & 0 & 0 & 23 & 0 & 3 & 0 & 0 & 0 & 0 & 7 & 0 & 0 & 0 & 33 & 7 & 0 & 0 & 63 & 139 \\
\hline \multicolumn{22}{|l|}{ Leptomeduea } \\
\hline Obelia sp. & 3 & 0 & 0 & 7 & 3 & 3 & 0 & 7 & 0 & 3 & 0 & 20 & 3 & 0 & 3 & 17 & 0 & 0 & 3 & 73 & 145 \\
\hline \multicolumn{22}{|l|}{ PTEROPODA } \\
\hline Cresis virgule & 0 & 0 & 0 & 0 & 0 & 0 & 0 & 0 & 33 & 0 & 3 & 0 & 20 & 0 & 3 & 0 & 50 & 0 & 10 & 0 & 119 \\
\hline Pteropod larvae & 0 & 0 & 0 & 10 & 0 & 0 & 0 & 10 & 0 & 0 & 0 & 33 & 0 & 0 & 0 & 117 & 0 & 0 & 0 & 117 & 287 \\
\hline \multicolumn{22}{|l|}{ CHAETOGNATHA } \\
\hline Krohnitta subtilis & 3 & 0 & 0 & 0 & 10 & 13 & 0 & 0 & 3 & 10 & 0 & 0 & 57 & 13 & 0 & 0 & 63 & 37 & 0 & 0 & 209 \\
\hline Sagita enflata & 0 & 0 & 0 & 0 & 0 & 0 & 0 & 0 & 10 & 0 & 0 & 0 & 50 & 0 & 0 & 3 & 107 & 0 & 0 & 3 & 173 \\
\hline \multicolumn{22}{|l|}{ UROCHORDATA } \\
\hline Appendicularia sicula & 7 & 3 & 0 & 3 & 7 & 0 & 3 & 17 & 3 & 13 & 7 & 7 & 13 & 10 & 10 & 27 & 10 & 17 & 10 & 3 & 170 \\
\hline Oikopleura longicauda & 23 & 0 & 23 & 0 & 57 & 0 & 23 & 3 & 167 & 30 & 33 & 43 & 233 & 33 & 33 & 83 & 317 & 50 & 100 & 107 & 1358 \\
\hline Doliolum denticullatum & 0 & 0 & 0 & 0 & 0 & 0 & 0 & 0 & 0 & 0 & 3 & 0 & 3 & 7 & 3 & 0 & 13 & 7 & 10 & 0 & 46 \\
\hline Polychaet larvae & 23 & 7 & 50 & 0 & 40 & 7 & 123 & 0 & 150 & 17 & 40 & 0 & 267 & 23 & 117 & 37 & 333 & 53 & 237 & 0 & 1524 \\
\hline Lamellibranch larvae & 180 & 0 & 0 & 0 & 113 & 0 & 0 & 0 & 107 & 3 & 0 & 0 & 83 & 0 & 0 & 0 & 100 & 7 & 0 & 0 & 593 \\
\hline Gastropod larvae & 277 & 97 & 43 & 20 & 277 & 33 & 73 & 57 & 720 & 33 & 83 & 17 & 617 & 380 & 33 & 30 & 667 & 267 & 33 & 150 & 3907 \\
\hline Cirripedia sp. & 7 & 40 & 183 & 57 & 7 & 43 & 217 & 57 & 17 & 150 & 187 & 50 & 27 & 180 & 190 & 167 & 30 & 173 & 57 & 140 & 1979 \\
\hline Cirriped nauplii & 0 & 10 & 0 & 0 & 0 & 7 & 0 & 0 & 0 & 13 & 0 & 0 & 0 & 0 & 0 & 0 & 0 & 0 & 0 & 0 & 30 \\
\hline Penaeid larvae & 17 & 0 & 0 & 0 & 10 & 3 & 0 & 0 & 0 & 0 & 0 & 0 & 3 & 0 & 0 & 0 & 43 & 0 & 0 & 0 & 76 \\
\hline Other decapod larvae & 7 & 0 & 0 & 23 & 17 & 0 & 0 & 10 & 3 & 0 & 0 & 40 & 23 & 0 & 0 & 33 & 17 & 0 & 0 & 30 & 203 \\
\hline Echinoderm larvae & 0 & 3 & 0 & 3 & 0 & 0 & 0 & 3 & 0 & 7 & 0 & 0 & 0 & 30 & 7 & 0 & 10 & 40 & 7 & 0 & 110 \\
\hline B. lanceolatum larvae & 0 & 0 & 0 & 0 & 0 & 0 & 0 & 0 & 0 & 0 & 0 & 0 & 0 & 0 & 0 & 17 & 0 & 0 & 0 & 17 & 34 \\
\hline Fish larvae & 17 & 0 & 33 & 3 & 30 & 7 & 50 & 10 & 27 & 20 & 0 & 0 & 30 & 117 & 3 & 0 & 37 & 17 & 3 & 20 & 424 \\
\hline Total & 1340 & 1920 & 1593 & 1803 & 2030 & 2250 & 2693 & 2277 & 3810 & 2853 & 3080 & 2310 & 3440 & 3197 & 4143 & 4023 & 6217 & 5367 & 6250 & 5703 & 66300 \\
\hline
\end{tabular}


Table 4. Significant differences of total zooplankton densities among different sites $(P \leq 0.01)$. (a,b,c,d and e refer to the significant differences).

\begin{tabular}{|l|c|c|c|c|c|}
\hline Sites*density & Site 1 & Site 2 & Site 3 & Site 4 & Site 5 \\
\hline Site 1 & - & $* *$ & $* *$ & $* *$ & $* *$ \\
\hline Site 2 & $* *$ & - & $* *$ & $* *$ & $* *$ \\
\hline Site 3 & $* *$ & $* *$ & - & $* *$ & $* *$ \\
\hline Site 4 & $* *$ & $* *$ & $* *$ & - & $* *$ \\
\hline Site 5 & $* *$ & $* *$ & $* *$ & $* *$ & - \\
\hline Sites*density & $6656 \mathrm{e}$ & $9250 \mathrm{~d}$ & $12053 \mathrm{c}$ & $14803 \mathrm{~b}$ & $23537 \mathrm{a}$ \\
\hline
\end{tabular}

\subsection{Frequency of zooplankton taxa}

The samples collected during the current study included 20 samples ( 4 seasons x 5 sites) from different locations in the studied area (Fig. 1). The constant taxa were 19 species, 5 larvae, Nauplius larvae and Copepodite stage, and the accessory taxa were 17 species and 2 larvae, while the rare taxa were 3 species, 2 larvae and Cirriped nauplii (Table 5).

Table 5. Frequency and percentage (\%) of different zooplankton taxa collected from the investigated sites during the period of collection.

\begin{tabular}{|c|c|c|c|c|c|c|c|c|c|c|c|c|}
\hline \multirow[b]{2}{*}{ Zooplankton taxa. } & \multicolumn{2}{|c|}{ Port-Tewfik } & \multicolumn{2}{|c|}{ Cabanon } & \multicolumn{2}{|c|}{ NIOF } & \multicolumn{2}{|c|}{ Ain Sokhna } & \multicolumn{2}{|c|}{ Zafarana } & \multicolumn{2}{|c|}{ all 5 sites } \\
\hline & $\mathbf{F}$ & $\%$ & $\mathbf{F}$ & $\%$ & $\mathbf{F}$ & $\%$ & $\mathbf{F}$ & $\%$ & $\mathbf{F}$ & $\%$ & $\mathbf{F}$ & $\%$ \\
\hline \multicolumn{13}{|l|}{ COPEPODA } \\
\hline Nauplius larvae & 4 & 100 & 4 & 100 & 4 & 100 & 4 & 100 & 4 & 100 & 20 & 100 \\
\hline Copepodite stage & 4 & 100 & 4 & 100 & 4 & 100 & 4 & 100 & 4 & 100 & 20 & 100 \\
\hline Paracalanus crassirostris & 4 & 100 & 4 & 100 & 4 & 100 & 3 & 75 & 4 & 100 & 19 & 95 \\
\hline Paracalanus parvus & - & 0 & - & 0 & 1 & 25 & 1 & 25 & 3 & 75 & 5 & 25 \\
\hline Paracalanus indicus & - & 0 & - & 0 & 1 & 25 & 1 & 25 & 3 & 75 & 5 & 25 \\
\hline Acrocalkanus gibber & 3 & 75 & 3 & 75 & 2 & 50 & 3 & 75 & 3 & 75 & 14 & 70 \\
\hline Centropages furcatus & 3 & 75 & 3 & 75 & 4 & 100 & 4 & 100 & 1 & 25 & 15 & 75 \\
\hline Acartia negligens & 4 & 100 & 4 & 100 & 4 & 100 & 3 & 75 & 3 & 75 & 18 & 90 \\
\hline Acartia centrura & 2 & 50 & 3 & 75 & 3 & 75 & 2 & 50 & 3 & 75 & 13 & 65 \\
\hline Clausocalanus furcatus & 3 & 75 & 2 & 50 & 3 & 75 & 2 & 50 & 4 & 100 & 14 & 70 \\
\hline Calanopia elliptica & 1 & 25 & 2 & 50 & 1 & 25 & 1 & 25 & 1 & 25 & 6 & 30 \\
\hline Calanus minor & 1 & 25 & 1 & 25 & 2 & 50 & 2 & 50 & 3 & 75 & 9 & 45 \\
\hline Mecynocera clause & - & 0 & 1 & 25 & 1 & 25 & 1 & 25 & 3 & 75 & 6 & 30 \\
\hline Oithona nana & 4 & 100 & 4 & 100 & 4 & 100 & 4 & 100 & 4 & 100 & 20 & 100 \\
\hline Oithona plumifera & 4 & 100 & 4 & 100 & 4 & 100 & 3 & 75 & 2 & 50 & 17 & 85 \\
\hline Oncaea media & 3 & 75 & 1 & 25 & 4 & 100 & 4 & 100 & 4 & 100 & 16 & 80 \\
\hline Oncaea conifer & - & 0 & - & 0 & - & 0 & - & 0 & 1 & 25 & 1 & 5 \\
\hline Corycaeus erythraeus & - & 0 & - & 0 & - & 0 & 1 & 25 & 3 & 75 & 4 & 20 \\
\hline Corycaeus speciosus & - & 0 & 1 & 25 & 1 & 25 & 2 & 50 & 3 & 75 & 7 & 35 \\
\hline Euterpina acutifrons & 3 & 75 & 3 & 75 & 3 & 75 & 2 & 50 & 3 & 75 & 14 & 70 \\
\hline Microsetella norvegica & 3 & 75 & 3 & 75 & 3 & 75 & 2 & 50 & 1 & 25 & 12 & 60 \\
\hline Clytemnestra scutellata & 2 & 50 & 2 & 50 & 2 & 50 & 1 & 25 & - & 0 & 7 & 35 \\
\hline Paracalanus aculatus & 2 & 50 & 2 & 50 & 2 & 50 & 1 & 25 & 1 & 25 & 8 & 40 \\
\hline Centropagus elongates & - & 0 & 2 & 50 & 2 & 50 & 2 & 50 & 3 & 75 & 9 & 45 \\
\hline
\end{tabular}




\begin{tabular}{|c|c|c|c|c|c|c|c|c|c|c|c|c|}
\hline Clausocalanus arcuicornisl & 1 & 25 & 1 & 25 & 1 & 25 & 2 & 50 & 3 & 75 & 8 & 40 \\
\hline Ctenocalanus vanus & - & 0 & 1 & 25 & 3 & 75 & 1 & 25 & 3 & 75 & 8 & 40 \\
\hline Farranula gibbula & - & 0 & 1 & 25 & 1 & 25 & 2 & 50 & 4 & 100 & 8 & 40 \\
\hline Copilia mirabilis & - & 0 & 1 & 25 & 3 & 75 & 1 & 25 & 1 & 25 & 6 & 30 \\
\hline \multicolumn{13}{|l|}{ TINTINNIDEA } \\
\hline Tintinnopsis campanula & 4 & 100 & 4 & 100 & 4 & 100 & 4 & 100 & 4 & 100 & 20 & 100 \\
\hline Favella ehrenbergii & 2 & 50 & 3 & 75 & 2 & 50 & 4 & 100 & 4 & 100 & 15 & 75 \\
\hline Stenosemella ventricosa & 1 & 25 & 2 & 50 & 4 & 100 & 3 & 75 & 3 & 75 & 13 & 65 \\
\hline \multicolumn{13}{|l|}{ FORAMINIFERA } \\
\hline Globigerina inflata & 4 & 100 & 4 & 100 & 4 & 100 & 4 & 100 & 4 & 100 & 20 & 100 \\
\hline \multicolumn{13}{|l|}{ Anthomedusea, } \\
\hline Podocoryne areolata & 1 & 25 & 2 & 50 & 1 & 25 & 1 & 25 & 2 & 50 & 7 & 35 \\
\hline \multicolumn{13}{|l|}{ Leptomeduea } \\
\hline Obelia sp. & 2 & 50 & 3 & 75 & 2 & 50 & 3 & 75 & 2 & 50 & 12 & 60 \\
\hline \multicolumn{13}{|l|}{ PTEROPODA } \\
\hline Cresis virgule & - & 0 & - & 0 & 1 & 25 & 2 & 50 & 2 & 50 & 5 & 25 \\
\hline \multicolumn{13}{|l|}{ CHAETOGNATHA } \\
\hline Krohnitta subtilis & 1 & 25 & 2 & 50 & 2 & 50 & 2 & 50 & 2 & 50 & 9 & 45 \\
\hline Sagita enflata & - & 0 & - & 0 & 1 & 25 & 1 & 25 & 2 & 50 & 4 & 20 \\
\hline \multicolumn{13}{|l|}{ UROCHORDATA } \\
\hline Appendicularia sicula & 3 & 75 & 3 & 75 & 4 & 100 & 4 & 100 & 4 & 100 & 18 & 90 \\
\hline Oikopleura longicauda & 2 & 50 & 3 & 75 & 4 & 100 & 4 & 100 & 4 & 100 & 17 & 85 \\
\hline Doliolum denticullatum & - & 0 & - & 0 & 1 & 25 & 3 & 75 & 3 & 75 & 7 & 35 \\
\hline Polychaet larvae & 3 & 75 & 3 & 75 & 3 & 75 & 4 & 100 & 3 & 75 & 16 & 80 \\
\hline Lamellibranch larvae & 4 & 100 & 1 & 25 & 2 & 50 & 1 & 25 & 2 & 50 & 10 & 50 \\
\hline Gastropod larvae & 4 & 100 & 4 & 100 & 4 & 100 & 4 & 100 & 4 & 100 & 20 & 100 \\
\hline Pteropod larvae & 1 & 25 & 1 & 25 & 1 & 25 & 1 & 25 & 1 & 25 & 5 & 25 \\
\hline Cirripedia sp. & 4 & 100 & 4 & 100 & 4 & 100 & 4 & 100 & 4 & 100 & 20 & 100 \\
\hline Cirriped nauplii & 1 & 25 & 1 & 25 & 1 & 25 & 0 & 0 & 0 & 0 & 3 & 15 \\
\hline Penaeid larvae & - & 0 & 2 & 50 & - & 0 & 1 & 25 & 1 & 25 & 4 & 20 \\
\hline Other decapod larvae & 2 & 50 & 2 & 50 & 2 & 50 & 2 & 50 & 2 & 50 & 10 & 50 \\
\hline Echinoderm larvae & 2 & 50 & 1 & 25 & 1 & 25 & 2 & 50 & 3 & 75 & 9 & 45 \\
\hline B. lanceolatum larvae & - & 0 & - & 0 & - & 0 & 1 & 25 & 1 & 25 & 2 & 10 \\
\hline Fish larvae & 3 & 75 & 4 & 100 & 2 & 50 & 3 & 75 & 4 & 100 & 16 & 80 \\
\hline
\end{tabular}

\subsection{Physico-chemical parameters}

The values of water temperature varied considerably between sites and seasons of investigation from winter 2018 to autumn 2019. The minimum water temperature was recorded in autumn at Port-Tewfik $\left(17.50^{\circ} \mathrm{C}\right)$, while the maximum value was recorded at Cabanon and NIOF in summer $\left(31^{\circ} \mathrm{C}\right)$. The minimum $\mathrm{pH}$ was recorded in winter at PortTewfik and in autumn at NIOF with 7.77, respectively, while the maximum value was recorded in autumn at NIOF (8.18). The minimum value of dissolved oxygen concentration was recorded in summer at NIOF $(5.10 \mathrm{mg} / \mathrm{l})$, while the maximum value 
was recorded in winter at Ain-Sokhna $(8.2 \mathrm{mg} / \mathrm{l})$. On the other hand, the minimum value of the total dissolved solids (T.D.S) concentration was recorded in autumn at AinSokhna, (36.30 g/l), while the maximum value was recorded in spring at Port-Tewfik (39.5 g/l) (Table 6).

Table 6. Mean \pm standard deviation $(\mathrm{SD})$ of physico-chemical parameters at the studied sites during different seasons.

\begin{tabular}{|c|c|c|c|c|c|}
\hline \multirow{2}{*}{ Sites } & \multirow{2}{*}{ Seasons } & W.Temp $\left({ }^{\circ} \mathrm{C}\right)$. & pH & $\mathrm{O}_{2}(\mathrm{mg} / \mathrm{l})$ & T.D.S (g/l) \\
\hline & & Mean \pm SD & Mean \pm SD & Mean \pm SD & Mean \pm SD \\
\hline \multirow{5}{*}{ a } & Winter & $19.00 \pm 0.5$ & $7.77 \pm 0.21$ & $6.80 \pm 0.21$ & $37.1 \pm 0.2$ \\
\hline & Spring & $23.50 \pm 0.26$ & $8.00 \pm 0.199$ & $6.00 \pm 0.19$ & $39.5 \pm 0.15$ \\
\hline & Summer & $28.00 \pm 0.26$ & $7.86 \pm 0.17$ & $6.10 \pm 0.02$ & $37.7 \pm 0.17$ \\
\hline & Autumn & $17.50 \pm 0.2$ & $7.87 \pm 0.11$ & $6.50 \pm 0.18$ & $36.7 \pm 0.21$ \\
\hline & Annual mean & $22 \pm 0.3$ & $7.8 \pm 0.17$ & $6.35 \pm 0.15$ & $37.75 \pm 0.18$ \\
\hline \multirow{5}{*}{ 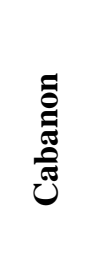 } & Winter & $20.00 \pm 0.36$ & $7.84 \pm 0.17$ & $7.80 \pm 0.05$ & $37.80 \pm 0.12$ \\
\hline & Spring & $25.50 \pm 0.26$ & $8.00 \pm 0.14$ & $5.60 \pm 0.034$ & $38.30 \pm 0.3$ \\
\hline & Summer & $31.00 \pm 0.46$ & $7.88 \pm 0.24$ & $5.30 \pm 0.085$ & $38.30 \pm 0.14$ \\
\hline & Autumn & $20.50 \pm 0.26$ & $7.75 \pm 0.07$ & $7.70 \pm 0.053$ & $37.90 \pm 0.19$ \\
\hline & Annual mean & $24.25 \pm 0.34$ & $7.87 \pm 0.16$ & $6.6 \pm 0.06$ & $38.08 \pm 0.19$ \\
\hline \multirow{5}{*}{$\frac{\sqrt[T]{x}}{2}$} & Winter & $20.00 \pm 0.5$ & $8.13 \pm 0.13$ & $6.20 \pm 0.18$ & $38.00 \pm 0.18$ \\
\hline & Spring & $26.00 \pm 0.56$ & $8.10 \pm 0.15$ & $5.50 \pm 0.105$ & $38.50 \pm 0.08$ \\
\hline & Summer & $31.00 \pm 0.2$ & $8.18 \pm 0.18$ & $5.10 \pm 0.12$ & $38.60 \pm 0.12$ \\
\hline & Autumn & $20.00 \pm 0.34$ & $7.77 \pm 0.07$ & $5.80 \pm 0.09$ & $38.60 \pm 0.25$ \\
\hline & Annual mean & $24.25 \pm 0.4$ & $8.045 \pm 0.13$ & $5.65 \pm 0.123$ & $38.4 \pm 0.16$ \\
\hline \multirow{5}{*}{ 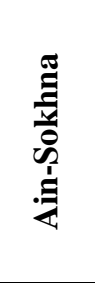 } & Winter & $20.50 \pm 0.36$ & $7.89 \pm 0.22$ & $8.20 \pm 0.04$ & $36.40 \pm 0.11$ \\
\hline & Spring & $25.00 \pm 0.36$ & $7.83 \pm 0.15$ & $6.10 \pm 0.12$ & $37.40 \pm 0.14$ \\
\hline & Summer & $30.50 \pm 0.26$ & $7.86 \pm 0.11$ & $6.30 \pm 0.17$ & $37.60 \pm 0.2$ \\
\hline & Autumn & $19.00 \pm 0.2$ & $7.93 \pm 0.15$ & $8.00 \pm 0.026$ & $36.30 \pm 0.13$ \\
\hline & Annual mean & $23.75 \pm 0.295$ & $7.88 \pm 0.16$ & $7.15 \pm 0.089$ & $36.9 \pm 0.15$ \\
\hline \multirow{5}{*}{ 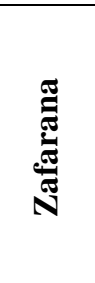 } & Winter & $18.50 \pm 0.17$ & $7.83 \pm 0.14$ & $7.80 \pm 0.03$ & $36.60 \pm 0.14$ \\
\hline & Spring & $24.50 \pm 0.44$ & $7.90 \pm 0.23$ & $6.20 \pm 0.105$ & $39.10 \pm 0.18$ \\
\hline & Summer & $29.50 \pm 0.36$ & $7.85 \pm 0.15$ & $5.50 \pm 0.26$ & $38.50 \pm 0.22$ \\
\hline & Autumn & $18.50 \pm 0.25$ & $7.88 \pm 0.07$ & $7.20 \pm 0.04$ & $36.50 \pm 0.19$ \\
\hline & Annual mean & $22.75 \pm 0.3$ & $7.87 \pm 0.15$ & $6.68 \pm 0.11$ & $37.68 \pm 0.18$ \\
\hline
\end{tabular}

By pooling data at the five sites and applying the correlation analysis between total numbers of zooplankton groups with physico-chemical parameters (ecological factors) concentrations in the seawater (Table 7), it was concluded that, the abundance of Tintinnidea was positively correlated with water temperature $(\mathrm{r}=0.456)$. To illustrate, the abundance of Anthomedusea was 
positively correlated with Leptomeduea $(\mathrm{r}=0.887)$ and negatively correlated with the total dissolved salts T.D.S $(r=-0.466)$. Furthermore, the abundance of Leptomeduea was positively correlated with Anthomedusea ( $r=0.887)$. While, the abundance of Pteropoda was positively correlated with Chaetognatha ( $\mathrm{r}=-$ 0.8), Uorochordata $(\mathrm{r}=0.89)$ and other larval stages $(\mathrm{r}=0.89)$. In addition, the abundance of Chaetognatha was positively correlated with dissolved oxygen $(\mathrm{r}=0.486)$, Uorochordata $(\mathrm{r}=0.868)$ and Pteropoda $(\mathrm{r}=0.89)$. While, the abundance of Uorochordata was positively correlated with dissolved oxygen $(\mathrm{r}=0.524)$, Pteropoda $(\mathrm{r}=0.89)$, Chaetognatha $(\mathrm{r}=0.868)$, while negatively correlated with the total dissolved salts $(\mathrm{r}=0.474)$ and the other larval stages $(\mathrm{r}=-$ 0.872). The abundance of Maxillopoda was positively correlated with water temperature $(r=0.635)$. It was also noted that, the abundance of other larval stages was positively correlated with dissolved oxygen $(\mathrm{r}=0.494)$, Pteropoda $(\mathrm{r}=0.846)$, Chaetognatha $(\mathrm{r}=0.778)$ and Uorochordata $(\mathrm{r}=0.872)$.

Table 7. The values of correlation coefficients ( $\mathrm{r}$ ) for different ecological parameters (Water Temperature (WT), Hydrogen Ion $(\mathrm{pH})$, Dissolved Oxygen $\left(\mathrm{O}_{2}\right)$, Total Dissolved Solids (T.D.S), Tintinidia (Tin), Formanifera (Form), Anthomedusea (Ant), Leptomeduea (Lep), Pteropoda (Pte), Chaetognatha (Cha), Copepoda (Cop), Uorochordata (Uro), Maxillopoda (Max), and Other larval species (Other).

\begin{tabular}{|c|c|c|c|c|c|c|c|c|c|c|}
\hline Factors & Tin. & Form. & Ant. & Lep. & Pte. & Cop. & Cha. & Uro & $\max$ & Other. \\
\hline & $(\mathrm{r})$ & (r) & $(\mathrm{r})$ & (r) & $(\mathrm{r})$ & (r) & (r) & (r) & (r) & (r) \\
\hline WT & $0.456^{*}$ & NS & NS & NS & NS & NS & NS & NS & $0.635 * *$ & NS \\
\hline $\mathrm{pH}$ & NS & NS & NS & NS & NS & NS & NS & NS & NS & NS \\
\hline $\mathrm{O}_{2}$ & NS & NS & NS & NS & NS & NS & $0.486^{*}$ & $0.524 *$ & NS & 0.494* \\
\hline T.D.S & NS & NS & $-0.466^{*}$ & NS & NS & NS & NS & $-0.474 *$ & NS & NS \\
\hline Tin & - & NS & NS & NS & NS & NS & NS & NS & NS & NS. \\
\hline Form & NS & - & NS & NS & NS & NS & NS & NS & NS & NS \\
\hline Ant & NS & NS & - & $0.887 * *$ & $\mathrm{NS}$ & NS & NS & NS & NS & NS \\
\hline Lep & NS & NS & $0.887 * *$ & - & NS & NS & NS & NS & NS & NS \\
\hline Pte & NS & NS & NS & NS & - & NS & $0.805 * *$ & $0.89 * *$ & NS & $0.846 * *$ \\
\hline Cop & NS & NS & NS & NS & NS & - & NS & NS & NS & NS \\
\hline Cha & NS & NS & NS & NS & $0.8^{* * *}$ & NS & - & 0.868 ** & NS & $0.778 * *$ \\
\hline Uro & NS & NS & NS & NS & $0.89 * *$ & NS & $0.868 * *$ & - & NS & $0.872 * *$ \\
\hline Max & NS & NS & NS & NS & NS & NS & NS & NS & - & NS \\
\hline Other. & NS & NS & NS & NS & $0.89 * *$ & NS & NS & $-0.872 *$ & NS & - \\
\hline
\end{tabular}

*: The mean difference is significant at the 0.05 levels.

**: The mean difference is significant at the 0.01 levels.

NS: the mean difference is not significant 


\section{DISCUSSION}

Zooplankton serves as an intermediate link between phytoplankton and higher consumers in both marine and freshwater ecosystems. Zooplankton providing food for larger animals and indirectly for humans through plankton-dependent fisheries, such as Sardines that feed on zooplankton in Egypt (Abou-Zeid, 1990). Because of their role in the marine food chain, zooplankton populations appear to be particularly helpful for assessing ecosystem health.

The current study showed spatial variations in zooplankton density and diversity among the surveyed sites. The maximum diversity was recorded from both Ain-Sokhna and Zafarana due to the lower pollution loads and the suitability of environmental conditions such as temperature and oxygen. While, the minimum diversity was recorded at Port-Tewfik due to the oil pollution from ships and less oxygen concentration, and the same conclusion was recorded in the study of Koppelmann et al. (2009). The statistical analysis evoked this finding, where there is a similarity (no significant differences) between three of the five investigated sites; NIOF, Ain-Sokhna and Zafarana, because they share similar conditions of low types of pollution, suitable ranges of temperature, the $\mathrm{pH}$ value and other environmental conditions. While, the other two sites (Port-Tewfik and Cabanon) showed significant differences in all the investigated sites due to the fact that, Port-Tewfik is oil polluted from ships' operations and human wastes, while Capanon is mainly affected by sewage pollution. The current finding agrees with that of Primo et al. (2015) who found that, the difference in the environmental conditions is of a prime importance causing the difference in zooplankton diversity, while Paturej et al. (2017), reported that anthropogenic activities greatly impact zooplankton diversity.

On contrary, the study revealed that all the sites investigated were significantly different in zooplankton density, which indicates that both environmental factors and human impacts have greater effect on density than diversity, these determined facts coincide with those of Thurman (1997), Brucet et al. (2010), Mitra et al. (2014) and Primo et al. (2015).

The current study recorded 16 taxa, and Copepods dominated the community structure of zooplankton in the study sites accounting for $79 \%$ of the total zooplankton sampled. This finding concurs with previous studies conducted in the Red Sea (Dorgham et al., 2012; Abu El-Regal et al., 2018), and Gulf of Aqaba (Michel et al., 1986; Dorgham \& Hussein, 1997). Additionally, Aboul Ezz et al. (2014) reported that, Copepods made up around $72 \%$ of the total zooplankton collected from Matrouh shore in the Mediterranean Sea. Copepods also dominated zooplankton communities in other regions, such as the Arctic (Greenland Sea, White Sea, Icelandic waters, Beaufort Sea, Kara Sea, Arctic Ocean) (Mumm, 1991; Richter, 1994; Auel \& Hagen, 2002; Fetzer et al., 2002; Walkusz et al., 2009; Dvoretsky \& Dvoretsky, 2013), whereas the 
Maxillopoda taxa was highly abundant; a phenomenon that may be attributed to being closer to the navigation route so the Cirripida larvae showed high abundance as a mean of biofouling.

The data obtained found that Oithona nana recorded the highest density during the current study from all sites that was attributed to their relatively lower metabolic rate and their wide-ranging dietary preferences (Lampitt \& Gamble, 1982; Castellani et al., 2005). Lampitt and Gamble (1982) classified the Oithona nana as a raptorial feeder, with an opportunistic diet able to consume particulates from detritus to phytoplankton including earlier stages of calanoid nauplii and even copepodite stages. The present result agrees with that of ObuidAllah et al. (2005), who found that the $O$. nana was the highest zooplankton species in the northern part of the Red Sea.

The study showed seasonal variations in zooplankton density at all study sites. The highest density was recorded in summer followed by spring, while it recorded the lowest density in winter. Notably, the suitable temperature for increasing densities of zooplankton species is $30^{\circ} \mathrm{C}$ which agrees with the result of Abu El-Regal et al. (2018). Hence, the reason for zooplankton peaked in summer is attributed to the suitable temperature, while the lowest temperature recorded from the area was $18.5^{\circ} \mathrm{C}$, that is why the lowest density was recorded in winter. These findings coincide with those of AlNajjar (2000) and Dorgham et al. (2012) in the Gulf of Suez and Abu El-Regal $\boldsymbol{e t}$ al. (2018) in the northern part of the Red Sea. On the contrary, the studies carried out at the Gulf of Aqaba recorded maximum density of zooplankton in winter (Echelman \& Fishelson, 1990; Khalil \& Abdel-Rahman, 1997). The density variations in zooplankton are affected by variations in the environmental conditions (Suresh $\boldsymbol{e t}$ al., 2011; Paturej et al., 2017).

\section{REFERENCES}

Abdel-Rahman, N. S. (1993). Ecological studies on the distriution of zooplankton communities in the northern part of the Suez Gulf (Suez Bay). M. Sc. Thesis, Dep. Zool. Fac. Sci., Suez Canal Univ., Egypt.

Abd El-Wakeil, K. F. (2005). Ecological studies on terrestrial Isopoda (Crustacea) in Assiut, Egypt. Ph.D. Thesis, Dep. Zool. Fac. Sci. Assiut Univ., Egypt.

Aboul Ezz, S.M.; Heneash, A.M.M. and Gharib, S.M. (2014). Variability of spatial and temporal distribution of zooplankton communities at Matrouh beaches, southeastern Mediterranean Sea, Egypt. Egypt. J. Aquat. Res., 40: 283-290.

Abou-Zeid, G.M. (1990). Distribution of zooplankton in Lake Timsah with special reference to Copepoda. M. Sc. Thesis, Dep. Fac. Sci. Suez Canal. Univ., Egypt.

Abu El-Regal, M.A; El-Wazeer, A.; Abou Elnaga, Z.S. and Amr, A.A. (2018). Composition and spatio temporal distribution of zooplankton community in the 
Egyptian Red Sea coast at Hurghada. Egyptian Journal of Aquatic Biology and Fisheries, 22(3): 1-12.

Al-Najjar, T.H. (2000). The seasonal dynamics and grazing control of Phytoand mesozooplankton in the northern Gulf of Aqaba, Ph.D. thesis, Bremen University.

Al-Yamani, F.Y. and V.A. Skryabin. (2006). Identification Guide for Protozoans from Kuwait's Waters. Coastal Planktonic Ciliates: 1.Tintinnids. Kuwait: Kuwait Institute for Scientific Research, 109 pp.

Al-Yamani, F.Y.; Khvorov, S.A. and Khvorov. A.S. (2010). Interactive Guide of Planktonic Decapod Larvae of Kuwait's Waters. Kuwait Institute for Scientific Researches. Electronic resource. Registry number: 2010/009 - 4, Kuwait.

Auel, H. and Hagen, W. (2002). Mesozooplankton community structure, abundance and biomass in the central Arctic Ocean, Mar. Biol., 140 (5): 1013-1021.

Bradford-Grieve, J.M. (1994). The marine fauna of New Zealand: pelagic calanoid Copepoda: Megacalanidae, Calanidae, Paracalanidae, Mecynoceridae, Eucalanidae, Spinocalanidae, Clausocalanidae. New Zealand Oceanogr. Inst. Mem., 102, 5-156.

Bradford-Grieve, J.M. and Jillet, J.b. (1980). The marine fauna of New Zealand: Pelagic calanoid copepods: family Aetideidae. Memoirs. N.Z Ocean. 1nst., 90: 1-150.

Brucet, S.; Boix, D.; Quintana, X.D.; Jensen, E.; Nathansen, L.W.; Trochine, C. and Jeppesena, E. (2010). Factors influencing zooplankton size structure at contrasting temperatures in coastal shallow lakes: implications for effects of climate change. Limnol. Oceanogr., 55(4): 1697-1711.

Castellani, C., Robinson, C., Smith, T. and Lampitt, R. S. (2005). Temperature affects respiration rate of Oithona similis. Mar. Ecol. Prog. Ser., 285, 129-135.

Cornils, A.; Schnack-Schiel, S.B.; Al-Najjar, T.; Rasheed, M.I.M.; Manasreh, R., and Richter, C. (2007). The seasonal cycle of the epipelagic mesozooplankton in the northern Gulf of Aqaba (Red Sea). Journal of Marine Systems: 278-292.

Dorgham, M.M. and Hussein, M.M. (1997). Zooplankton dynamics in a neritic area of the Arabian Gulf (Doha Harbour), Arab Gulf J. Scient. Res., 15 (2): 415-435.

Dorgham, M.M.; Elsherbiny, M.M. and Hanafi, M.H. (2012). Vertical distribution of zooplankton in the epipelagic zone off Sharm El-Sheikh, Red Sea, Egypt. Oceanologia, 54 (3): 473-489.

Dvoretsky, V.G. and Dvoretsky, A.G. (2013). Summer mesozooplankton community of Moller Bay (Novaya Zemlya Archipelago, Barents Sea). Oceanologia, 55: 205218.

Echelman, T. and Fishelson, L. (1990). Surface zooplankton dynamics and community structure in the Gulf of Aqaba (Eilat), Red Sea. Marine Biology, 107: 179- 190

El-Sherbiny, M.M.; Hanafy, M.H. and Aamer, M.A. (2007). Monthly variations in abundance and species composition of the epipelagic zooplankton off Sharm ElSheik, Northern Red Sea. Res. J. Environ. Sci., 1: 200-210. 
Fetzer, I.; Hirche H.J. and Kolosova, E.G. (2002). The influence of freshwater discharge on the distribution of zooplankton in the southern Kara Sea, Polar Biol., 25 (6): 404-415. http://dx.doi.org/10.1007/s00300-001-0356-5.

Gatenby, J.B. and Painter, T.S. (1937). A handbook of the methods of animal and plant microscopic anatomy (the microtomist's vade mecum). Philadelphia PA: P. Blakiston's Son and Co.

Heron, G.A. and Bradford-Grieve, J.M. (1995). The marine fauna of New Zealand: Pelagic Copepoda: Poecilostomatoida: Oncaeidae (Vol. 104). New Zealand Oceanographic Institute.

Kaya, M.; Fontaneto, D.; Segers, H. and A tındağ, A. (2010). Temperature and salinity as interacting drivers of species richness of planktonic rotifers in Turkish continental waters. J. Limnol., 69: 297304.

Khalil M.T. and Abdel-Rahman N.S. (1997). Abundance and diversity of surface zooplankton in the Gulf of Aqaba, Red Sea, Egypt, J. Plankton Res., 19: 927-936.

Koppelmann, R.; Böttger-Schnack, R.; Möbius, J. and Weikert, H. (2009). Trophic relationships of zooplankton in the eastern Mediterranean based on stable isotope measurements. Journal of Plankton Research, 31(6): 669-686,

Lampitt, R.S. and Gamble, J.C. (1982) Diet and respiration of the small planktonic marine copepod Oithona nana. Mar. Biol., 66: 185-190.

Michael, P. (1984). Ecological methods for field and laboratory investigations. Tata Megraw-Hill Publishing Company Limited, New Delhi, First Edition, 404 pp.

Michel, H.B.; Behbehani, M. and Herring, D. (1986). Zooplankton of the Western Arabian Gulf South of Kuwait waters, Kuwait Bull. Mar. Sci., KISR, Ser. No. 1435: 1-36.

Mitra, A.; Castellani, C.; Gentleman, W.C.; Jónasdóttir, S.H.; Flynn, K J. and Bode, A. (2014). Bridging the gap between marine biogeochemical and fisheries sciences; configuring the zooplankton link. Progress in Oceanography, 129: 176-199.

Mumm N. (1991). On the summer distribution of mesozooplankton in the Nansen Basin, Arctic Ocean, Ber. Polarforsch, 92: 1-173.

ObuidAllah, A.H.; Abdallah, A.T.; Abu-Eldahab, H.M.; Abdul-Rahman, N. S. and Mahdy, A. (2005). Impact of heavy metal contamination on seasonal abundance of planktonic copepods inhabiting mangrove area in Safaga, Red Sea, Egypt. Egypt. J. Exp. Biol, 1, 123-130.

Paturej, E.; Gutkowska, A.; Koszałka, J. and Bowszys, M. (2017). Effect of physicochemical parameters on zooplankton in the brackish, coastal Vistula Lagoon, Oceanologia, 59: 49- 56.

Primo, A.; Kimmel, D.; Marques, S.; Martinho, F.; Azeiteiro, U. and Pardal, M. (2015). Zooplankton community responses to regional-scale weather variability: a synoptic climatology approach. Clim. Res., 62(3): 189-198. 
Raitsos, D.E.; Pradhan, Y.; Brewin, R.J.; Stenchikov, G. and Hoteit, I. (2013). Remote sensing the phytoplankton seasonal succession of the Red Sea. PloS one, 8(6), e64909.

Richter, C. (1994). Regional and seasonal variability in the vertical distribution of mesozooplankton in the Greenland Sea, Ber. Polarforsch., 154: 1-90.

Sofianos, S.S. and Johns, W.E. (2002). An oceanic general circulation model (OGCM) investigation of the Red Sea circulation, 1. Exchange between the Red Sea and the Indian Ocean. Journal of Geophysical Research: Oceans, 107(C11):1-17.

Sparks, T. (2000): Statistics in Ecotoxicology. John Wiley \& Sons Ltd.

Suresh, S.; Thirumala, S. and Ravind, H.B. (2011). Zooplankton diversity and its relationship with physicochemical parameters in Kundavada Lake, of Davangere District, Karnataka, India. ProEnvironment 4 (7): 56- 59.

Thurman, H.V. (1997): Introductory oceanography. New Jersey: Prentice Hall.

Walkusz, W.; Kwaśniewski, S.; Falk-Petersen, S.; Hop, H.; Tverberg, V.; Wieczorek, P. and Węsławski, J.M. (2009). Seasonal and spatial changes in the zooplankton community in Kongsfjorden, Svalbard, Polar Res., 28 (2): 254-281. http: //dx.doi.org/10.1111/j.1751-8369.2009.00107.x.

Wyatt, A.S.J.; Falter, J.L.; Lowe, R.J.; Humphries, S. and Waite, A.M. (2012). Oceanographic forcing of nutrient uptake and release over a fringing coral reef. Limnology and Oceanography, 57: 401- 419. 